\title{
EchoGéo
}

$28 \mid 2014$

Police : les espaces de l'ordre, l'ordre en espace

\section{Le gendarme de Saint-Astier : les dispositifs spatiaux dédiés au maintien de l'ordre}

Anthony Goreau-Ponceaud et Émilie Ponceaud-Goreau

\section{OpenEdition}

1 Journals

Édition électronique

URL : https://journals.openedition.org/echogeo/13753

DOI : $10.4000 /$ echogeo.13753

ISSN : 1963-1197

Éditeur

Pôle de recherche pour l'organisation et la diffusion de l'information géographique (CNRS UMR 8586)

Référence électronique

Anthony Goreau-Ponceaud et Émilie Ponceaud-Goreau, « Le gendarme de Saint-Astier : les dispositifs spatiaux dédiés au maintien de l'ordre », EchoGéo [En ligne], 28 | 2014, mis en ligne le 08 juillet 2014, consulté le 31 juillet 2021. URL : http://journals.openedition.org/echogeo/13753 ; DOI : https://doi.org/ 10.4000/echogeo.13753

Ce document a été généré automatiquement le 31 juillet 2021.

EchoGéo est mis à disposition selon les termes de la licence Creative Commons Attribution - Pas d'Utilisation Commerciale - Pas de Modification 4.0 International (CC BY-NC-ND) 


\title{
Le gendarme de Saint-Astier : les dispositifs spatiaux dédiés au maintien de l'ordre
}

\author{
Anthony Goreau-Ponceaud et Émilie Ponceaud-Goreau
}

\section{Introduction}

1 Saint-Astier, commune de plus de 5000 habitants au cœur de la vallée de l'Isle, située à 18 kilomètres à l'Ouest de Périgueux (qui abrite une école nationale de police) est marquée par deux structures nettement identifiables dans le paysage et qui en font sa notoriété: les fours à chaux ${ }^{1}$ et le centre national d'entraînement des forces de gendarmerie ou CNEFG. C'est ce deuxième élément qui retiendra ici notre réflexion et qui capte également l'attention des nouveaux arrivants ou gens de passage qui manifestent leur surprise lorsqu'ils croisent, devant le supermarché, des blindés ${ }^{2}$ montant vers le plateau de Crognac, ou encore lorsqu'ils observent une épaisse fumée noire s'échappant des hauteurs de la ville. Il faut dire qu'à Saint-Astier, les forces de l'ordre ont produit leur géographie: un espace à l'écart de la ville où il s'agit de s'entraîner au maintien de l'ordre. Véritable emprise spatiale, le CNEFG puise ses origines dans les événements de mai $1968^{3}$ lorsque la nécessité d'assurer l'entraînement et le perfectionnement des escadrons de gendarmerie mobile ${ }^{4}$ au maintien de l'ordre est apparue de manière éloquente aux opérateurs politiques. Le traumatisme de mai 68 a eu d'importantes répercussions sur la redéfinition du maintien de l'ordre: des stratégies en vigueur aux techniques de contrôle social en passant par l'équipement, l'armement et les renseignements des gendarmes mobiles.

2 «Tenir la rue » (Fillieule, 1997) est une dimension substantielle de l'action de l'État et donne tout son sens à la formule de Bayley: «la légitimité du pouvoir est largement dépendante de sa capacité à maintenir l'ordre. L'ordre est même le critère pour dire si un pouvoir politique existe ou non » (Bayley 1985, p. 5). Avec la police, la gendarmerie est la seule administration susceptible d'intervenir partout et à n'importe quel moment 
pour obtenir le retour à une situation considérée comme normale, et ce travail policier à la fois exprime cette normalité sociale et l'impose concrètement dans le quotidien. Le recours à la rue comme médium de contestation est devenu de plus en plus banal. Selon Fillieule et Della Porta, "très logiquement, cette routinisation s'accompagne d'une pacification marquée des conflits» (Della Porta, Fillieule, 2006, p. 12). C'est à SaintAstier que les unités de gendarmerie mobile s'entraînent et se perfectionnent aux techniques du maintien de l'ordre. Cette acquisition de techniques soulève un faisceau de questions auxquelles cet article tentera d'apporter des réponses: quels sont les fondements des doctrines et des pratiques policières contemporaines en matière d'ordre public? Existe-t-il des modèles spatiaux de gestion des conflits sociaux? Quelles sont les stratégies, le vocabulaire et les tactiques policières?

3 La compréhension du maintien de l'ordre, comme l'a souligné Olivier Fillieule (1997), ne peut se limiter à l'étude des seules pratiques et attitudes policières, mais doit intégrer celles des manifestants. C'est pourquoi, il se joue à Saint-Astier de manière régulière, une théâtralisation des "violences urbaines " ${ }^{5}$ reposant sur des jeux de rôles où tour à tour les gendarmes deviennent des faux manifestants et les faux manifestants des gendarmes.

Espace à l'écart de la ville, et ville à l'écart, cet « espace-écart » (Doraï et Puig, 2012, p. 19), cette scène de théâtre si particulière, nous a semblé être un terrain privilégié pour rendre compte d'une production spatiale de l'ordre. Notre démonstration s'appuie sur des entretiens semi-directifs menés au sein du CNEFG auprès de deux officiers ${ }^{6}$. Notre démonstration s'organisera en deux temps. Dans un premier temps, nous présenterons l'emprise spatiale du CNEFG et les stratégies de maintien de l'ordre, avant d'analyser, dans un second temps, la manière dont l'espace a été modélisé par les instructeurs du CNEFG pour former à l'intervention professionnelle ${ }^{7}$ et au maintien de l'ordre. 


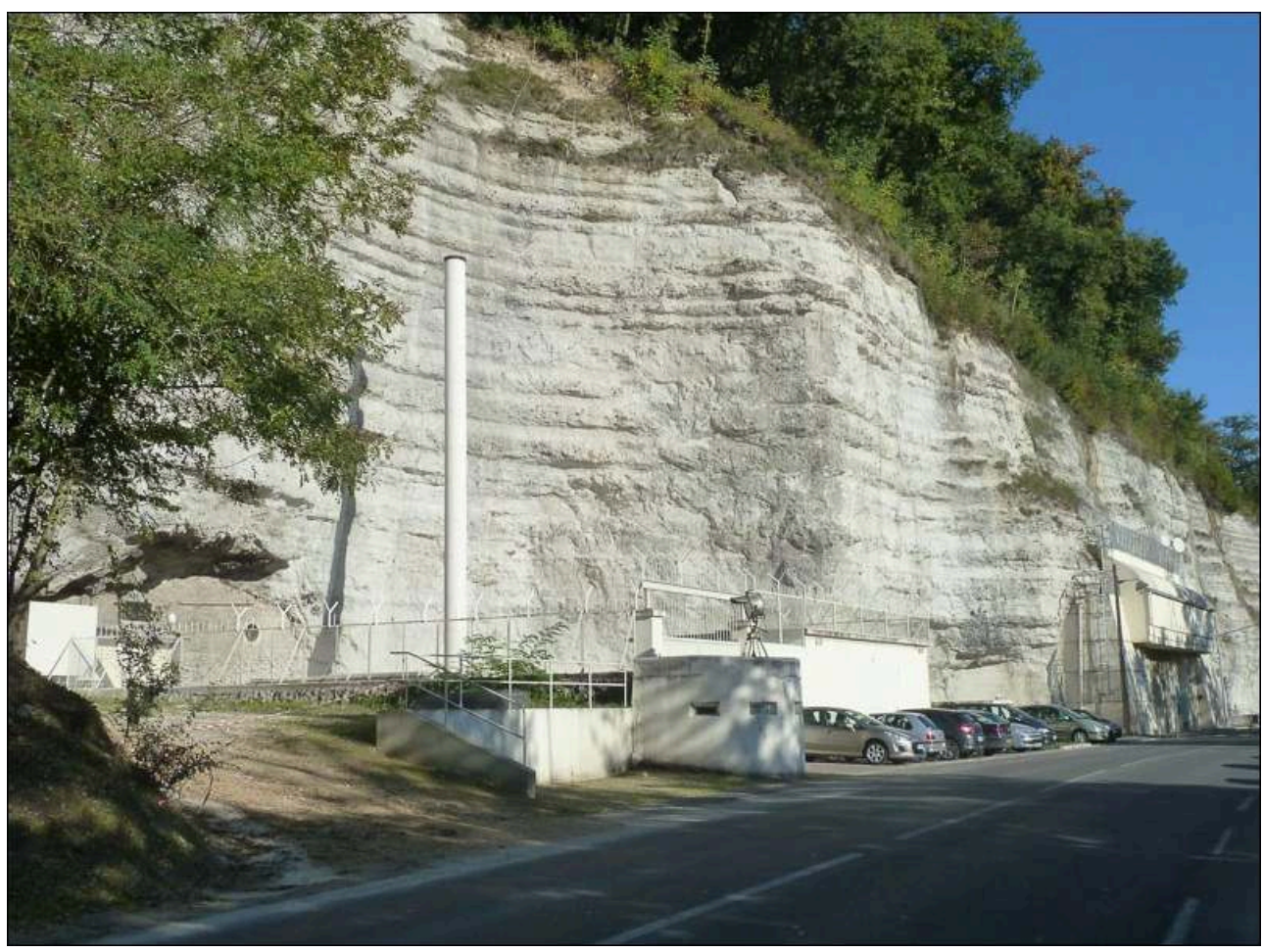

Au premier plan la RD3. Creusés dans les falaises calcaires, les anciens fours à chaux qui hébergent le $13^{\text {ème }}$ BS MAT. Sur les hauts, le CNEFG.

Auteurs : A. Goreau-Ponceaud et É. Ponceaud-Goreau, février 2014.

\section{Le CNEFG : une emprise spatiale au cœur des stratégies de rétablissement de l'ordre}

5 Profitant du départ des aviateurs de la base aérienne de soutien de Saint-Astier (BA 276) et appuyés par quelques politiques locales comme Yves Guéna - ministre des postes et télécommunications à l'époque et député de la Dordogne - les premiers instructeurs du Centre de Perfectionnement de la Gendarmerie Mobile (CPGM) prennent possession du plateau de Crognac le $1^{\mathrm{er}}$ avril 1969. Au cours de ses 44 années d'existence, le centre connaît diverses appellations et formats ${ }^{8}$. Depuis 1999, il porte le nom de CNEFG. Ce centre accueille la formation au rétablissement de l'ordre depuis 1969. Le retour d'expériences de l'engagement de la gendarmerie mobile lors des évènements de mai 68 a fait apparaitre la nécessité de créer un centre d'expertise, garant de l'unicité de la doctrine, au profit de l'ensemble des escadrons de gendarmerie mobile.

\section{Une emprise spatiale}

6 Le centre forme une emprise spatiale de 140 hectares située sur les hauteurs de la ville. La Caserne Général Dupuy se compose :

- de structures d'accueil et de soutien des stagiaires (pouvant accueillir environ 600 stagiaires). 
- d'installations d'entraînement (tour d'escalade ou tour d'initiation aux techniques d'intervention, stand de tir...),

- de plusieurs parcours d'audace individuel et collectif,

- d'une ville artificielle d'exercices. Elle sert à l'entraînement des forces de gendarmerie mobile dans des situations de maintien ou de rétablissement de l'ordre.

- d'un cinéma où sont projetés des films fabriqués par la cellule audiovisuelle et d'infographie du centre.

- le soutien logistique qui comprend entre autres un état-major, un service de restauration, du matériel et un garage automobile et une antenne médicale composée de trois médecins et trois infirmiers. Tous les gendarmes de la Dordogne et le personnel du service militaire adapté de Périgueux viennent ici pour la médecine du travail et s'y faire soigner. Le CNEFG possède également quelques équipements sportifs : un gymnase, une salle de musculation et un dojo où on y enseigne la maîtrise sans armes de l'adversaire.

7 Chaque année le centre reçoit plus de 13000 stagiaires. Tous ces stages sont encadrés par des officiers et sous-officiers d'expérience, spécialistes du maintien de l'ordre ou de l'intervention professionnelle. Le Centre compte environ 210 officiers, sous-officiers, gendarmes adjoints et personnels civils. Ces derniers représentent près du tiers des effectifs et permettent de développer un lien avec la société locale, au-delà de l'enclosure du terrain militaire. Ce lien est également entretenu par les contacts et les divers partenariats qu'a su nouer le CNEFG avec les élus, la municipalité ou encore le collège. Le bénéfice du centre pour la ville de Saint-Astier est direct et continu depuis sa création. Néanmoins, le turn-over des gendarmes reste important ${ }^{9}$ car il est nécessaire que les instructeurs repartent régulièrement sur le terrain.

\section{Le maintien de l'ordre}

Constitue un attroupement tout rassemblement de personnes sur la voie publique ou dans un lieu public susceptible de troubler l'ordre public.

Un attroupement peut être dissipé par la force publique après deux sommations de se disperser demeurées sans effet, adressées par le préfet, le sous-préfet, le maire ou l'un de ses adjoints, tout officier de police judiciaire responsable de la sécurité publique, ou tout autre officier de police judiciaire, porteurs des insignes de leur fonction.

Il est procédé à ces sommations suivant des modalités propres à informer les personnes participant à l'attroupement de l'obligation de se disperser sans délai.

Toutefois, les représentants de la force publique appelés en vue de dissiper un attroupement peuvent faire directement usage de la force si des violences ou voies de fait sont exercées contre eux ou s'ils ne peuvent défendre autrement le terrain qu'ils occupent.

Les modalités d'application des alinéas précédents sont précisées par décret en Conseil d'Etat, qui détermine également les insignes que doivent porter les personnes mentionnées au deuxième alinéa et les conditions d'usage des armes à feu pour le maintien de l'ordre public (Article 431-3 du code pénal).

Le CNEFG est le point de passage obligé de toutes les formations au maintien de l'ordre public. Cette unicité de formation assure la cohérence du dispositif global dans le respect du cadre juridique du maintien de l'ordre qui est celui de la dispersion des attroupements (article 431-3 du code pénal). Cette cohérence permet également aux moblos de bénéficier d'un avantage, l'expertise ; une ressource qui permet de s'assurer dans la manifestation de la domination des groupes peu expérimentés et de les amener à coopérer dans le sens souhaité. Ajoutons que la manière dont le maintien de l'ordre 
s'effectue en réalité ne saurait s'analyser uniquement à travers l'arsenal législatif, la gendarmerie, à l'instar de la police s'appuie sur des moyens de négociations (Fillieule, 2006, p. 94-95).

Le maintien de l'ordre public doit permettre de faire coexister deux libertés apparemment contradictoires, à savoir la liberté de manifestation qui est perçue comme une facette de la liberté d'expression et la préservation de la paix publique. Depuis la création des premières unités mobiles, il semble que la pacification du maintien de l'ordre soit une constante. Si Donatella Della Porta, Abby Peterson et Herbert Reiter (2013) ont récemment avancé que les protestations de 1968 auraient conduit les polices occidentales à développer des modes plus pacifiés de maintien de l'ordre, et fondés sur le dialogue voire une relative tolérance aux désordres, notre position converge davantage avec le constat émis par Bruneteaux (1996) et Mathieu (2013) : ce processus de pacification est, en France, antérieur à mai 1968, qui n'a fait que le renforcer en le "sophistiquant» (Bruneteaux, 1996, p. 27). La sophistication des moyens et des nouvelles techniques d'intervention a ainsi permis de développer le concept de "distanciation » entre manifestants et forces de l'ordre pour obtenir une économie de la violence. Partant du principe que le contact est la principale cause d'incidents violents, il s'agit avant tout pour les forces de l'ordre de maintenir à distance le manifestant/adversaire à travers divers moyens (barrières, projectiles lacrymogènes,...) et diverses techniques (manœuvre). L'utilisation du terme adversaire n'est pas neutre: elle fait exister la norme en même temps qu'elle exprime la construction de typologies et de profils de manifestants de la part des moblos, qui dès lors émettent des jugements de déviance. Pour les moblos:

«L'ennemi est hors des frontières, à l'intérieur il n'y a que des "adversaires » auxquels on laissera toujours, quelle que soit la rudesse de l'affrontement, une porte de dispersion, une voie de retraite » (entretien du 16/10/13).

C'est donc la sophistication de la gestion du rapport entre proximité et distance qui est au cœur de la pacification du maintien de l'ordre. Cette technicisation de la « lutte des places » (Lussault, 2009) repose sur le double principe de l'acceptation d'un désordre raisonnable et de la professionnalisation des forces en charge du maintien de l'ordre public. Ainsi, la conduite des opérations combine appui et mouvement, en appliquant, dans le cadre de la loi, les principes de gradation et de proportionnalité dans l'intervention. Le gendarme doit faire preuve d'un usage modéré des pouvoirs que lui confèrent la loi et les règlements en recherchant la négociation en permanence avec les manifestants (gradation). Les gendarmes mobiles utilisent un niveau de force proportionné au trouble à réprimer et la force déployée prend fin lorsque l'élément déclencheur cesse (proportionnalité). 
Illustration 2 - Le principe de gradation

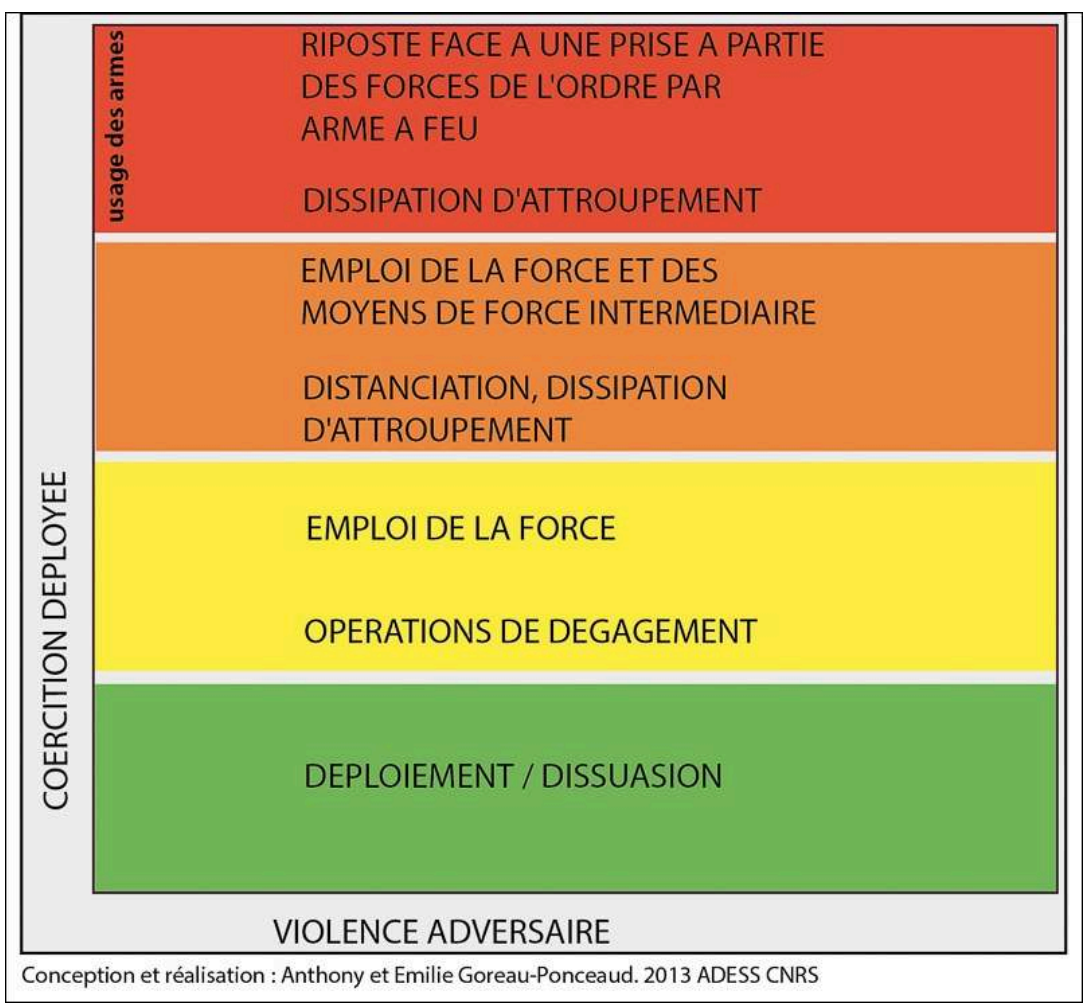

Selon une étude menée par Fillieule, « interrogés sur ce qui leur paraît le moins acceptable de subir de la part des forces de l'ordre, les manifestants 'préfèrent' être chargés (22 \%) qu'être soumis à des tirs de bombes lacrymogènes (8\%) ou des lanceurs d'eau (4\%) » (Fillieule,2006, p. 103). On le voit, du côté des forces du maintien de l'ordre, cette gradation suit un ordre inverse, posant de fait la question des perceptions de la pacification du maintien de l'ordre.

11 Cependant, l'évolution des actions des manifestants ainsi que le développement des moyens de communication modernes et les réseaux sociaux permettant le rassemblement d'un grand nombre de personnes, sans préavis, en déjouant les contraintes d'autorisation, ont conduit les gendarmes mobiles à modifier leurs dispositifs souvent monolithiques et à appliquer un principe de modularité.

«Les mouvements, les manifestations sont de plus en plus diffus, mobiles et éclatés. Les regroupements se recomposent très vite avec les nouvelles technologies de l'information et des communications. De même, ces dernières années nous n'étions plus confrontés à des opérations de maintien de l'ordre en zone rurale. Depuis les grandes manifestations anti-nucléaires à Golfech(31) ou encore Creys-Malville(38), il n'y avait quasiment eu que des interventions en zone urbaine. Le cas récent de Notre-Dame-desLandes a nécessité la mise en cuvre d'un nouvel exercice d'entraînement spécifique aux modes opératoires à adopter dans ce nouvel espace d'emploi » (entretien du 16/10/13).

Dans cette complexification des mouvements sociaux, le téléphone portable semble représenter un outil perturbant, d'une part, parce qu'il trouble les stratégies de positionnements des gendarmes mobiles, les manifestants pouvant s'agréger et se disperser rapidement dans un même cadre espace-temps ; et d'autre part, parce que les manifestants ont le réflexe de prendre des photographies, ce qui génère une tension supplémentaire pour les gendarmes mobiles qui font face à l'exigence des citoyens dans un contexte de judiciarisation généralisé.

«Les adversaires, avec leur téléphone portable dernière génération, ont le réflexe de saisir en photo ou en vidéo tout évènement particulier ou susceptible d'alimenter la controverse. C'est 
pourquoi nous nous équipons aussi de technologies similaires à la fois pour mener des interpellations mais également pour prévenir ce type de controverse. Un maintien de l'ordre est très sensible par définition. Les conséquences peuvent en être très importantes et engager directement la responsabilité de l'exécutif. » (entretien du 16/10/13).

13 Les gendarmes mobiles doivent adapter leurs stratégies de maintien de l'ordre à celles des manifestants. Dans cette perspective, le CNEFG constitue un laboratoire permettant d'expérimenter non seulement des modes d'actions nouveaux mais également les futurs matériels de la gendarmerie mobile avant leur adoption éventuelle.

\section{Un espace de formation au maintien de l'ordre : la ville d'exercices}

14 Au cœur de l'emprise spatiale constituée par le CNEFG, une pensée de l'espace s'est peu à peu structurée à travers une géographie empirique des corps en mouvement. Pour penser les corps en mouvement mais également le mouvement de leur propre corps (positionnement adéquat face à une situation de danger, gestes à adopter) lors de manifestations, d'attroupements, d'émeutes ou encore lors de violences urbaines, les instructeurs du CNEFG ont progressivement produit une modélisation de l'intervention professionnelle et du maintien de l'ordre.

\section{Un espace écart}

«Ce décor a été conçu en fonction d'une sédimentation de connaissances empiriques accumulées au fil des années et en prenant en compte les évolutions des comportements des adversaires » (entretien du 02/10/13).

Dès le milieu des années 1970, le CNEFG se démarqua des autres centres d'entraînement par la création d'une ville dans la ville. En effet, la spécificité du centre tient à ce que la ville d'entraînement n'est pas un espace urbain préexistant mais a été construit ex nihilo. Il s'agit plus exactement d'une ville d'exercices qui est utilisée pour la formation au maintien de l'ordre et à l'intervention professionnelle. La construction de ce décor ne s'est pas faite à partir d'un plan existant. L'espace a été conçu et les aménagements pensés par les instructeurs qui y ont progressivement apporté leur savoir à partir du terrain. En cela cette ville prend des allures de contre-espace sur une superficie de 27 hectares. Plus précisément, "Cigaville » comme certains l'appelaient au moment du service militaire a tout d'une hétérotopie (Foucault, 2009) : un lieu hors de tous lieux ménagé par la société dans ses marges, dont l'utilité est de rétablir une normalité selon des critères fixés par l'État. Il s'agit pour les moblos de modéliser le maintien de l'ordre et de comprendre la manière dont les mobilisations, parfois brutales, se créent dans un espace donné, et surtout la manière dont elles sont façonnées par l'espace dans lequel elles émergent et se déploient.

Ce contre-espace est l'expression de la « virtualisation » de l'entraînement, c'est-à-dire l'association d'un savoir géographique théorique (lire des cartes, utiliser la géolocalisation, utiliser le vocabulaire géographique) et d'un savoir pratique dont l'apprentissage se fait dans des espaces virtuels. Si cette pensée spatiale s'inspire largement de la pensée géographique militaire plus traditionnelle (qu'elle soit tactique ou stratégique) revendiquée dès l'Art de la guerre de Sun Tzu et, de façon plus contemporaine, par Yves Lacoste, elle s'en distingue par sa virtualité. 
Partant de l'idée que «l'espace et le lieu déterminent, tout en les favorisant, l'émergence des mouvements de contestation » (Auyero, 2005, p. 126), les gendarmes mobiles s'exercent à actualiser les potentiels qu'offre l'espace tout en le restructurant. De façon continuelle lors des exercices, les instructeurs agissent sur l'organisation matérielle de l'espace pour agir sur la mobilisation protestataire. Le plus grand réalisme est recherché dans le montage des exercices de rétablissement de l'ordre. Au cœur de ces exercices, l'élément central est le scénario concernant le dispositif spatial de l'attroupement et sa gestion par les forces du maintien de l'ordre. Un gendarme, au sommet de la tour de surveillance, donne des ordres, contrôle, juge de l'efficacité et fait évoluer le scénario. Dès lors, se met en place un véritable jeu de plateforme où le but est de contrôler le déplacement des manifestants.

Illustration 3 - La ville d'entraînement ou pôle 1

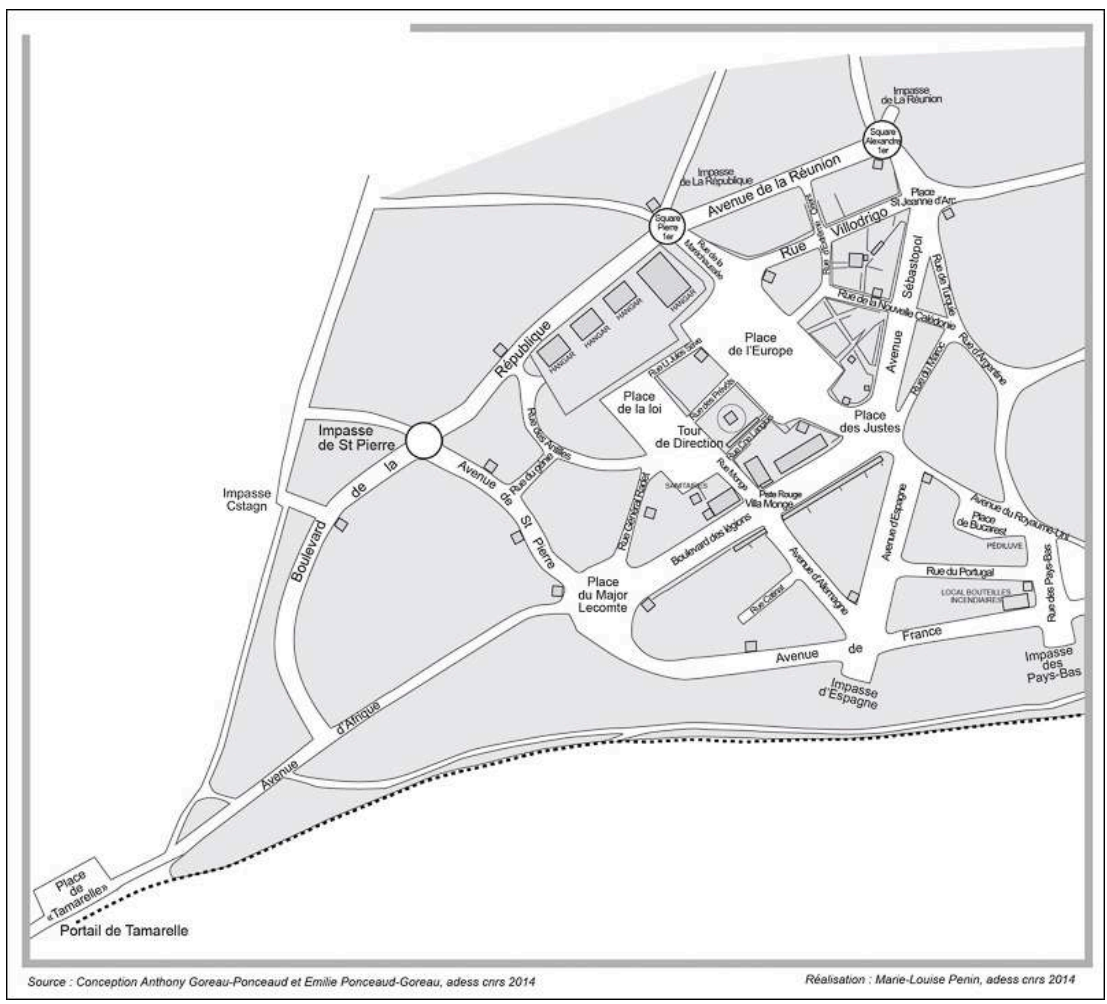

Les noms de rue, avenue, place et impasse de la ville d'exercice reprennent soit des noms de grands corps de l'armée, soit des noms de pays ou de régions associés aux actions de formation du CNEFG. 


\section{Illustrations 4a - Le Boulevard des légions de jour lors d'un exercice de rétablissement de l'ordre}

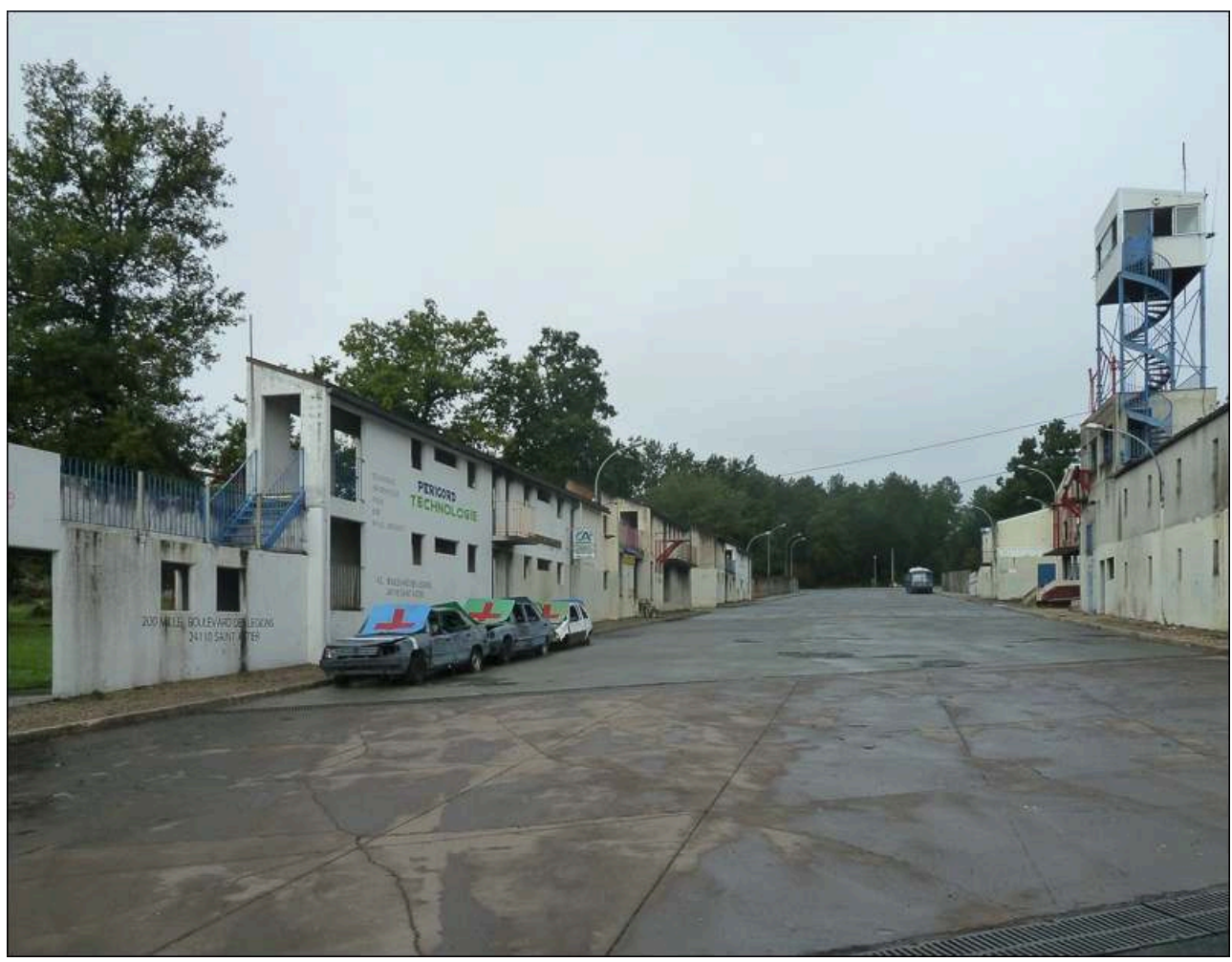

Les voitures, les bus sont déplacés au grès du bon vouloir du responsable de l'exercice afin de tester la réactivité des gendarmes. Ce dernier, du haut de sa tour, visible à droite de la photo, fait évoluer le scénario et contrôle ses « avatars » qui doivent contraindre le rétablissement du maintien de l'ordre. A la demande, certains manifestants peuvent changer de comportement et d'autres, repérables à leur plastron de couleur vive, viennent compliquer les manœuvres en utilisant divers artefacts. La menace de débordement est en cours et les moblos doivent la maîtriser. La violence, comme dans l'espace public, est le fait des manifestants et de l'État. Cet espace, du fait de sa modularité/virtualité, est le support de dispositifs spatiaux qui produisent, en retour, des comportements spatiaux, le temps d'un scénario. Ce que l'on peut noter dans ces jeux de rôle c'est une habitude de la part des cadres du CNEFG de l'usage des corps comme force physique dans le cadre d'actions radicales. À l'inverse on note l'absence d'une pensée spatiale de l'usage symbolique et médiatique des corps.

Auteurs : A. Goreau-Ponceaud et E. Ponceaud-Goreau, 2014. 
Illustration $4 b$ - Le Boulevard des légions de nuit lors d'un exercice de rétablissement de l'ordre

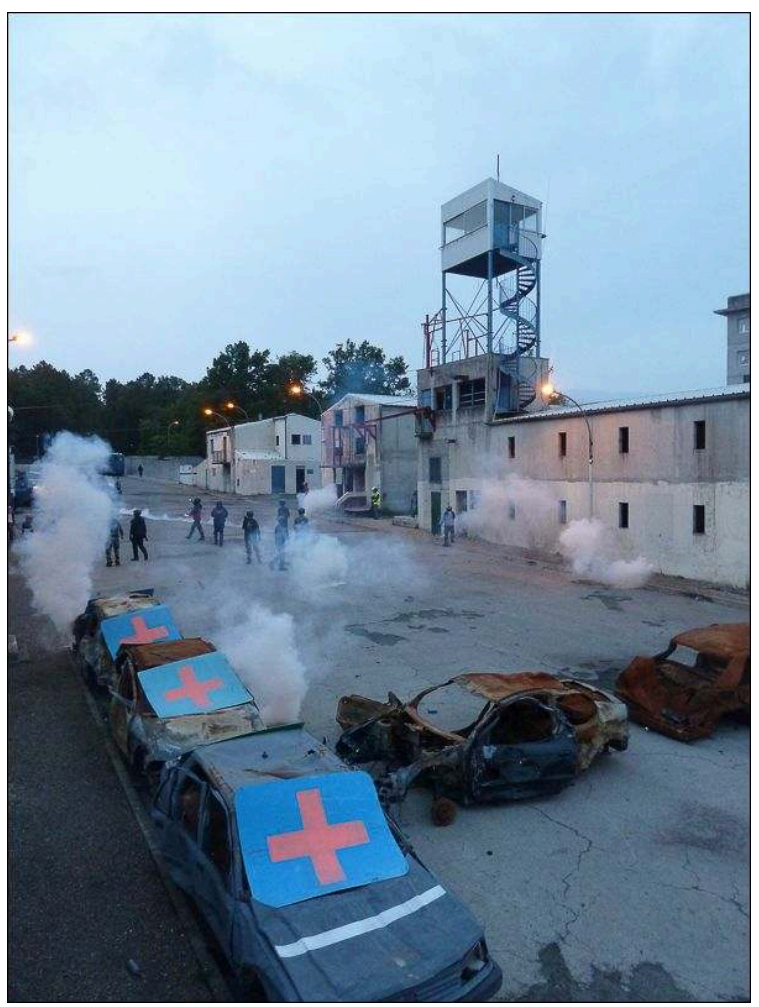

Auteurs : A. Goreau-Ponceaud et E. Ponceaud-Goreau, 2014

18 L'espace de simulation est un espace à trois dimensions: c'est d'abord un espace physique (un décor modulable selon les scénarios), un espace sonore (l'ambiance sonore est omniprésente et le bruit assourdissant) et enfin un espace évolutif dans le temps (mise en place de barricades, évolution des rôles de certains personnages, jet de grenades lacrymogènes et de bombes incendiaires...).

"Le fond sonore diffusé par les haut-parleurs est assourdissant. Il faut imaginer que le chef militaire doit analyser la situation dans le bruit, de nuit, avec un masque à gaz, avec le risque de ne pas être entendu ou mal compris. L'intensité des exercices est bien supérieure à la plupart des opérations réelles. Généralement, sur six unités présentes, quatre sont forces de l'ordre, deux jouent les manifestants. Par convention de manœuvre, les manifestants non casqués sont considérés comme calmes; le fait de mettre le casque indique une montée de la violence du côté des plastrons. Une équipe d'animation est chargée de mettre en place des barricades et d'incendier des véhicules stationnés ou composant la barricade. L'attroupement se transforme progressivement en violences urbaines " (entretien du 02/10/13). 
Illustration 5 - La ville lors d'un exercice nocturne de rétablissement de l'ordre

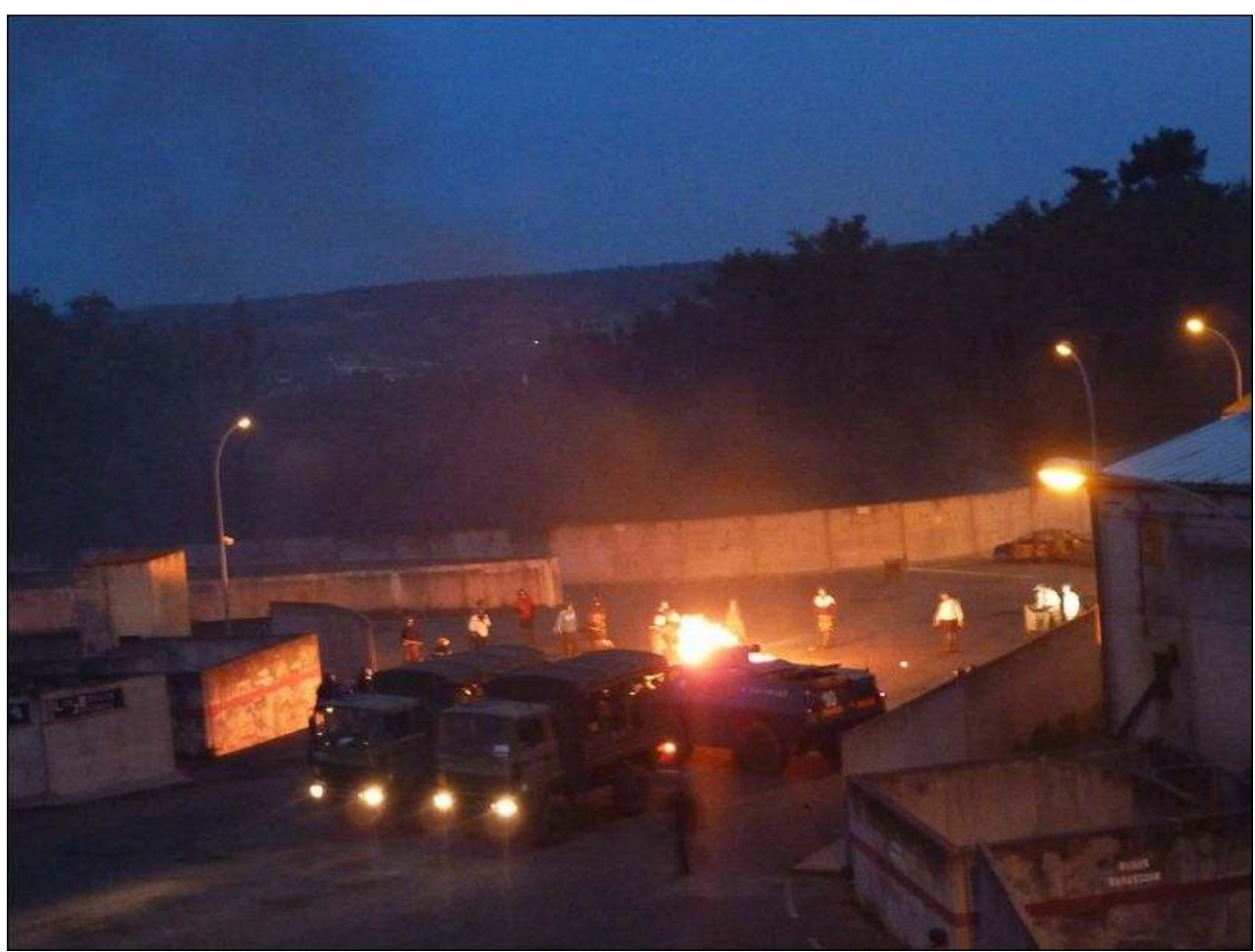

Le 3 juin 2014, le CNEFG a conduit un exercice nocturne de rétablissement de l'ordre. Six escadrons provenant de Chambéry, Belfort, St-Nazaire, Le Havre, Lodève et Maisons-Alfort, composés de quatre pelotons chacun, représentant un total de 402 hommes ont joué le scénario d'une opposition intertribale en outre-mer (Nouvelle-Calédonie). Trois escadrons engagés comme forces de l'ordre sous le commandement d'un lieutenant-colonel commandant le groupement de gendarmerie tactique (GTG) de Mont-de-Marsan et les trois autres jouant les plastrons. Des jeunes de tribus différentes se sont opposés, certains ont été blessés. La gendarmerie a procédé à l'interpellation de trois individus pour mettre un terme à leurs agissements. Leurs tribus sont venues manifester aux portes de la brigade pour qu'on les libère. Les missions lors de ce scénario sont multiples : escorter les pompiers sur un incendie de véhicule, les accompagner et les faire sortir du dispositif, faire face à des exactions dans la ville, évacuer un blessé par balle au sein des forces de l'ordre par hélicoptère, localiser et interpeller le tireur isolé, puis contenir, repousser, disperser la foule lors du transport des trois individus interpellés au palais de justice. Accompagnant ce dispositif : le SDIS de Périgueux, des véhicules blindés à roues de la gendarmerie (VBRG) du CNEFG pilotés par des personnels du groupement blindés de la gendarmerie mobile (GBGM) de Versailles-Satory, un hélicoptère EC 135 de la SAG de Toulouse (équipé d'une caméra thermique) en appui aérien, une équipe médicale opérationnelle de la gendarmerie (EMOG). Ce scénario renvoie notamment aux événements récents qui se sont déroulés à Saint-Louis, localité de la commune de Mont-Dore (proche de Nouméa), connue pour être le lieu de nombreux affrontements violents entre communautés mélanésiennes de la tribu et wallisiennes du lotissement voisin de l'Ave Maria. En effet, le 25 mai 2014, deux gendarmes ont été blessés par balles sur la RP1, suite à une situation sociale extrêmement tendue nécessitant la mobilisation des gendarmes mobiles.

Auteurs : A. Goreau-Ponceaud et E. Ponceaud-Goreau, Place de l'Europe - ville d'exercice -vers 22h30, le 3 juin 2014.

19 On assiste également à une évolution des tactiques spatiales d'intervention, du statique au dynamique :

«L'adversaire n'est plus le même, on est passé des grosses manifestations avec un effet de masse important, pour lequel on utilisait des barrages d'arrêts fixes, beaucoup de statique, on refoulait, on scindait, on déplaçait... Le déploiement ne se fait plus de la même façon qu'il y a 15 ans. Avant, pour un escadron (environ 65 personnes déplacées sur une mission), on faisait 3 pelotons de 25 dans 3 bus. Aujourd'hui, l'escadron est partagé en 4 pelotons qui se déplacent grâce à 8 petits véhicules mobiles. $\mathrm{Au}$ sein $\mathrm{du}$ pôle 1 , plusieurs thèmes sont joués: les 
manifestations, les violences urbaines, et également des éléments spécifiques à l'outre-mer comme l'affrontement entre deux communautés, comme ce que l'on a pu rencontrer en Nouvelle Calédonie. [...] » (entretien du 16/10/13). largement la construction d'une géographie imaginée. Ces représentations poussent les moblos à développer des typologies d'adversaires dont on peut s'attendre à ce qu'ils occasionnent des difficultés. L'action des moblos dépend ainsi des ordres reçus mais également de l'image qu'ils se font des groupes eux-mêmes. Précisons que la formation est également un temps où vont se forger des représentations, des classements explicites des groupes de manifestants et une typologie dans les répertoires de l'action non institutionnelle. C'est aussi durant ce temps de formation que semble se mettre en place une perception de la légitimité des groupes à être présents dans la rue et à avoir recours à tel ou tel mode d'action : les moblos apprennent ici à faire une «distinction entre bons et mauvais manifestants » (Fillieule, 2006, p. 108) ce qui laisse supposer une hétérogénéité dans les opérations de maintien de l'ordre.

21 Par ailleurs, cet espace d'exercices est un ensemble modulaire comprenant plusieurs éléments qui représentent des géotypes (cf illustration 3 ) :

- la ville, avec ses rues étroites (rue des Antilles, rue de la Nouvelle Calédonie), ses avenues (avenue d'Espagne, avenue d'Allemagne...), ses larges places (place de l'Europe, des Justes...);

- la barre, véritable attribut et synecdoque du grand-ensemble, où l'architecte baigné d'une pensée fonctionnaliste a segmenté les espaces empêchant ainsi toute fluidité dans l'action du maintien de l'ordre (où le confinement constitue dès lors un piège pour le gendarme) ;

- des espaces plus lâches à l'urbanisation diffuse : le périurbain.

Un hameau composé de 4 maisons sert de décor aux scénarios ruraux. A l'inverse, un bus est utilisé dans le but de travailler les déplacements confinés. Le centre souhaiterait acquérir un wagon et construire un morceau de quai pour former au mieux les agents de la SUGE ${ }^{10}$ qu'il accueille actuellement.

«Zone urbaine, périurbaine et rurale: il s'agit de trois types de zones bien distinctes. La manœuvre en zone rurale n'a rien à voir avec la manifestation de rue ou l'occupation de site industriel. Dans les grands ensembles comme dans les zones urbaines, il faut prendre en considération la hauteur des bâtiments, l'existence des balcons. Des objets arrivent de très haut parfois. On a construit ce genre de bâtiment pour faire travailler les gendarmes dans des conditions réalistes. On intègre aussi les halls d'immeuble, on a fait des caves et des souterrains pour apprendre à conduire des interpellations ou chercher des éléments matériels dans ce genre d'endroits. On peut aussi monter sur les toits. Le centre s'est construit en copiant ce que l'on rencontre en architecture sur le terrain et intègre des thèmes d'exercices s'inspirant de cas concrets rencontrés au quotidien. Il faut meubler ${ }^{11}$ à la réalité. Dans la ville d'exercice, il y a de larges avenues, d'autres moins importantes, des rues étroites, de grandes places... En bref, l'environnement urbain que l'on connaît [...]. La grande tour là haut, c'est la tour d'exercice ; c'est de là que le directeur d'exercice déroule le scénario de l'exercice » (entretien du 02/10/13).

Le centre (le pôle 1) s'est donc construit en copiant ce à quoi les gendarmes sont confrontés comme éléments architecturaux, comme forme urbaine, tout en intégrant dans cette matérialité des thèmes d'exercices en accord avec les types de délinquance auxquels ils font le plus régulièrement face. Des éléments se sont ajoutés, comme en 2008, avec la création d'un espace de simulation qui fait penser à une rue commerçante (illustration 5) constituée d'un bar (le bar de la menace), d'une poste, d'une "superette ", d'un guichet de retrait d'argent automatique, et d'un hôtel. Une partie seulement du 
réseau viaire a été constituée comprenant des passages piétons et trois entrées possibles sur la rue (qui constituent de multiples configurations pour les scénarios d'interpellation). Tous ces espaces bâtis (les espaces pleins de cet espace de simulation) sont meublés pour prendre en considération les éléments qui freinent l'avancée des gendarmes. Dans cet espace, les gendarmes se forment à l'IP et sont observés par des caméras. Une passerelle située au-dessus de ce décor permet aux instructeurs d'observer le mouvement des corps et l'emplacement des gendarmes dans ce dispositif spatial.

\section{Illustration 6 - L'espace de simulation}

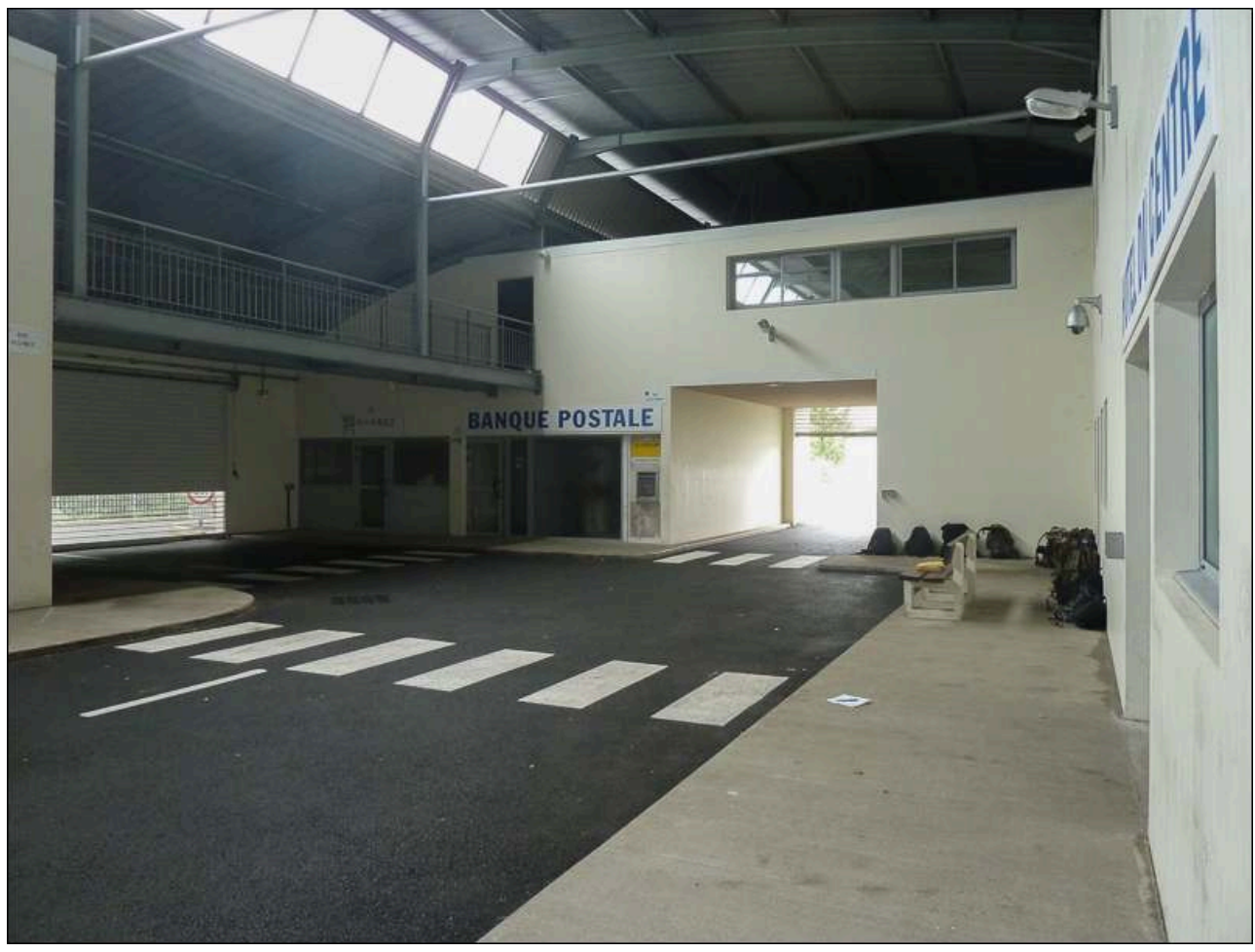

L'usage de dispositifs virtuels, que ce soit des simulateurs ou ce type d'espace, n'est pas neuf, et l'armée américaine a largement contribué à développer des outils d'entraînement de plus en plus sophistiqués, qui font la part belle à la mise en situation, sous forme de jeu de rôle (Halter, 2006). Lorsque nous avons mené nos entretiens, à plusieurs reprises dans la conversation entre les officiers, il a été question d'insérer des nouvelles technologies dans les scénarios (drone notamment).

«Il s'agit de travailler la tactique de l'intervention et le positionnement précis de celui qui contrôle un véhicule ou de celui qui interpelle un individu à pied, ou qui progresse à l'intérieur d'un appartement... Ces gendarmes seront des moniteurs d'IP ou des instructeurs d'IP et auront pour mission de relayer ces savoir-faire auprès des personnels de la compagnie voire du groupement d'appartenance, permettant aujourd'hui «d'innerver » l'institution toute entière » (entretien du $16 / 10 / 13)$.

\section{Un vocabulaire géographique efficace}

La progression du gendarme sur son lieu d'exercice ou au centre se fait à travers les spécificités du milieu en utilisant le discours géographique: territoire d'action, localisations, déplacements, positions, mise en avant de réseaux. 

ambition de questionner la dimension spatiale des stratégies et tactiques du maintien de l'ordre et de leur progressive virtualisation. Nous avons pu constater que le CNEFG constitue un centre d'expertise garant de l'unicité de la doctrine et des dispositifs spatiaux concernant les stratégies de maintien et de rétablissement de l'ordre. Les instructeurs y ont progressivement produit une pensée spatiale de la «lutte des places » (Lussault, 2009) qui s'exporte produisant in extenso une uniformisation dans l'organisation des polices nationales en matière d'ordre public. 
«La semaine prochaine nous accueillons au centre le ministre de l'intérieur du Pérou. Nous envoyons des instructeurs pour mener à bien des opérations de formation dans plus de cinquante pays par an. Nous avons participé à la reconstruction de la gendarmerie en Roumanie et nous effectuons des exercices tactiques avec la plupart des forces de l'ordre en Europe. Dernièrement la Jordanie nous a sollicités-pour l'aider à former une gendarmerie (à quelques kilomètres au Sud d'Amman). Actuellement elle est constituée de 23000 hommes et nous allons envoyer des formateurs là-bas. C'est très important, ce sera le premier modèle du genre dans la région. La première fois que s'y implante cette "troisième force " comme la nomment les anglo-saxons : une force de police à statut militaire aux capacités intermédiaires entre une police classique et les capacités de puissance de l'armée de terre. Vous savez le modèle de gendarmerie s'exporte très bien » (entretien du 16/10/13).

Les échanges qui lient le centre à d'autres pays ont tendanciellement pour effet d'uniformiser tout à la fois les modes organisationnels (par exemple, la nature des liens hiérarchiques, la différentiation entre types de force et types de mission, la formation des hommes), les doctrines d'emploi, les savoirs professionnels et les pratiques routinières dans une perspective d'interopérabilité et de transférer ce modèle de troisième force, entre police et armée. Cette uniformisation allant au-delà des sociétés occidentales ${ }^{13}$. Néanmoins, dans ce centre d'entraînement se construit aussi une représentation de la rue, de ceux qui sont légitimes pour tenir la rue et une construction de la démocratie qui repose sur des logiques binaires ${ }^{14}$. Ce sont ces mêmes logiques et ces mêmes représentations du bon et du mauvais manifestant qui sont exportées. En somme comme le mentionne Ripoll, "tout se passe comme si la manifestation, ou toute action de masse, s'inscrivait bel et bien dans le paradigme démocratique, mais au cœur d'une tension paradoxale entre deux de ses modalités d'existence : la représentation et la participation directe » (Ripoll, 2008, p. 91).

\section{BIBLIOGRAPHIE}

Auyero J., 2005. L'espace des luttes. Topographie des mobilisations collectives. Actes de la recherche en sciences sociales, 2005/5, $\mathrm{n}^{\circ} 160, \mathrm{p} .122-132$.

Bayley D., 1985. Patterns of Policing. A Comparative International Analysis. New Brunswick, Rutgers University Press, 280 p.

Bruneteaux, P, 1996. Maintenir l'ordre. Les transformations de la violence d'État en régime démocratique. Paris, Presses de Sciences-Po, 352p.

Della Porta D., Fillieule O. (dir.), 2006. Police et manifestants. Maintien de l'ordre public et gestion des conflits. Paris, Presses de Sciences-Po, $362 \mathrm{p}$.

Della Porta D., Peterson A. \& Reiter, H. (dir.), 2013. The policing of transnational protest. Farnham, Ashgate, $224 \mathrm{p}$.

Doraï, K., Puig N., 2012. Un lointain si proche. L'urbanité des marges. Migrants et réfugiés dans les villes du Proche-Orient. Paris, Téraèdre/IFPO, $335 \mathrm{p}$. 
Favre P (dir.), 1990. La manifestation. Paris, Presses de Sciences-Po, 392 p.

Fillieule O, 2006. Du pouvoir d'injonction au pouvoir d'influence ? Les limites de l'institutionnalisation. In Della Porta D., Fillieule O (dir.), Police et manifestants. Maintien de l'ordre public et gestion des conflits. Paris, Presses de Sciences-Po, p. 85-112.

Fillieule O, 1997. Stratégies de la rue. Paris, Presses de Sciences-Po, 440 p.

Foucault M., 2009. Le corps utopique, les hétérotopies. Clamecy, Nouvelle éditions Lignes, 61 p.

Halter E., 2006. From Sun Tzu to Xbox: war and videogames. New York, Thunder's Mouth Press, 364 p.

Lussault M., 2009. De la lutte des classes à la lutte des places. Paris, Editions Grasset, 220 p.

Mathieu L., 2013. L'autre côté de la barricade : perceptions pratiques policières en mai et juin 1968. Revue historique, vol. 1, nº65, p. 145-172.

Mathieu L., 2011. Territoires et mises en espace des mobilisations. In Bonny Y, Ollitrault S., Keerle R., Le Caro Y., Espaces de vie, espaces enjeux. Entre investissements ordinaires et mobilisations politiques. Rennes, Presses Universitaires de Rennes, p. 185-195.

Mucchielli L., 2000. L'expertise policière de la « violence urbaine », sa construction intellectuelle et ses usages dans le débat public français. Déviance et société, vol. 24, n 4, p. 351-375.

Ripoll F., 2008. Espaces et stratégies de résistance : répertoires d'action collective dans la France contemporaine. Espaces et sociétés, ${ }^{\circ} 134$, p. 83-97.

\section{NOTES}

1. Le calcaire du crétacé supérieur de Saint-Astier lui assure sa réputation mondiale par l'exploitation de la chaux, et certaines carrières exploitées à la fin du XIXe siècle, sont devenues des entrepôts militaires, à l'abri des regards, accueillant le régiment du matériel : le 13ème BS MAT.

2. Le Groupement Blindé de Gendarmerie Mobile (GBGM) organise parfois des exercices interarmées au CNEFG dont les scénarios sont aussi divers que l'évacuation, sur un théâtre extérieur, de ressortissants français pris en otage à l'Ambassade de France et la récupération d'autres nationaux éparpillés sur zone (exercice mené en 2010 par exemple visant la préparation des unités aux opérations extérieures, ou OPEX).

3. Comme le précise Mathieu (2013, p 145) : «Plus de quarante ans après, la manière dont les pratiques policières ont pesé sur mai 68 reste un enjeu majeur pour la compréhension des événements, et cela d'autant plus que leur perception a connu de sensibles fluctuations ». Mai 1968 a, en quelque sorte, constitué pour les forces de l'ordre une épreuve dont il a fallu tirer les leçons.

4. La Gendarmerie Mobile est une subdivision d'armes de la gendarmerie nationale française spécialisée dans le maintien et le rétablissement de l'ordre public. La création des unités de gendarmerie mobile remonte à 1921. Elle se compose de 108 escadrons de gendarmerie mobiles sur l'ensemble du territoire, soit pour chaque escadron, environ 100 personnes. La spécificité de la gendarmerie mobile tient en ce que, contrairement aux autres forces de maintien de l'ordre, comme les CRS, elle est régulièrement envoyée pour servir outre-mer, ou dans le cadre d'opérations extérieures. En effet, la gendarmerie mobile renforce les unités territoriales des gendarmes existants sur l'ensemble des événements qui se déroulent sur la métropole, les DOM TOM et à l'extérieur comme en Afghanistan, en Côte d'Ivoire et au Kosovo dernièrement. Sur le territoire national, cela peut être la sécurisation d'événements type G8, G20, la venue de personnalités ou des rendez-vous sportifs de grande importance (Tour de France, match de 
football). Sans connotations péjoratives, les gendarmes mobiles sont plus communément appelés, les « Moblos ».

5. Au-delà des idéologies partisanes et des clivages gauche/droite, l'expression violence urbaine renvoie à la montée du thème sécuritaire dans l'agenda politique (thème souvent au centre des campagnes électorales) et au durcissement de la politique de l'immigration. Selon Mucchielli, la généalogie de ce terme est à relier à la mise en place d'une nouvelle expertise policière, d'un nouveau discours qui «énonce une double présomption de culpabilité et de dangerosité des jeunes d'origine maghrébine » (Mucchielli, 2000, p. 369). Néanmoins, la gendarmerie n'est pas la police et même si cette troisième force reste attentive aux besoins politiques du maintien de l'ordre, elle semble moins enfermée dans des logiques corporatives et sensible aux mutations sociales (c'est du moins ce qui transparaît au cours de nos entretiens).

6. Nous avons choisi de rendre anonyme les propos de ces deux officiers pour deux raisons : cela nous permet d'échapper à une relecture de la part des instances dirigeantes de la gendarmerie nationale et donc de maintenir une distance critique nécessaire au regard des points de vue exprimés dans les extraits d'entretien présentés dans le corps du texte.

7. L'intervention professionnelle ou IP. L'IP représente l'ensemble des tactiques ou des techniques « simples » individuelles et collectives, applicables par tous les gendarmes, destinées à contraindre ou maîtriser un individu avec le strict niveau de force nécessaire. Cela fait partie du tronc commun à tous les gendarmes.

8. En 1971, le CPGM devient le Centre d'Instruction de la Gendarmerie (CIG), il assure ainsi la formation d'élèves-gendarmes issus des gendarmes auxiliaires. En 1973, le centre devient le Centre d'Instruction des Gendarmes Auxiliaires (CIGA). Sa fonction est d'assurer la formation initiale des appelés du contingent servant en gendarmerie avant leur affectation en unités de gendarmerie départementale. En 1977, le CPGM, inactif depuis 1971, est recréé sous le nom de Division de Perfectionnement de la Gendarmerie Mobile (DPGM). En 1984, le centre prend le nom d'École de Sous-Officiers de Gendarmerie - Centre d'instruction de gendarmes auxiliaires de Saint-Astier Bergerac - (ESOG-CIGA). En 1999, l'école devient le Centre National d'Entrainement des Forces de Gendarmerie (CNEFG) et la DPGM devient la Direction de l'Instruction (DI).

9. Les officiers et les sous-officiers ne restent sur le centre qu'une courte période: 4 ans pour les premiers, 5 ans pour les autres.

10. La SUrveillance GEnérale composée d'agents de la SNCF.

11. Nous trouvons cette expression employée par le capitaine très pertinente et révélatrice de la pensée "géographisante » menée par le CNEFG. Les éléments du pôle 1 constituent les meubles d'un décor qu'il s'agit d'agencer en fonction de la récurrence de certaines configurations manifestantes. Par ailleurs, les immeubles sont également meublés pour intégrer des réflexes liés au positionnement du corps, à l'alignement des bras, au maintien de l'arme.

12. Equipe Régionale d'Intervention et de Sécurité.

13. Selon les personnes interrogées le 16/10/13 cette uniformisation : " C'est aussi une manière d'exporter notre conception des libertés publiques et des droits de l'homme ».

14. On peut rappeler à titre d'illustration les propos tenus par Michèle Alliot-Marie le 12 janvier 2011, en proposant, à l'Assemblée nationale, le savoir-faire français à la police tunisienne pour "régler les situations sécuritaires" (Le Monde, édition du 13 janvier 2011). 


\section{RÉSUMÉS}

À partir d'entretiens et d'observations menés au Centre National d'Entraînement des Forces de Gendarmerie (CNEFG) à Saint-Astier, cet article a pour ambition d'analyser la manière dont se sont progressivement structurées une pensée de l'espace et une modélisation du maintien de l'ordre public. Plus particulièrement, il s'agit de comprendre de quelle manière les gendarmes mobiles produisent des dispositifs spatiaux pour contrôler les corps en mouvement lors d'attroupements, de manifestations et de «violences urbaines». Notre analyse porte ainsi un intérêt particulier sur un espace singulier du centre : la ville d'exercices où les instructeurs au maintien de l'ordre bouleversent l'organisation matérielle de l'espace pour agir sur la mobilisation protestataire.

This paper aims to analyze the way public forces have designed a dedicated space to simulating maintenance of public order. This research is based on interviews and observations conducted at the National Training Center of the French Gendarmerie forces at Saint-Astier. More specifically, the point is to understand how the anti-riot gendarmes keep under control the bodies during gatherings, protests and "urban riots". Our analysis focuses on the training area which is a space aside.

\section{INDEX}

Mots-clés : gendarme mobile, dispositif spatial, manifestation, ordre, espace, ville Keywords : Anti-riot gendarmes, spatial monitoring, space, order, city

\section{AUTEURS}

\section{ANTHONY GOREAU-PONCEAUD}

Anthony Goreau-Ponceaud, anthonygoreau@yahoo.fr, est docteur en géographie, membre de l'UMR ADESS CNRS, enseignant chercheur à l'université de Bordeaux. Il a publié récemment : - Goreau-Ponceaud A., 2014. Ganesha Chaturthi and the Sri Lankan Tamil Diaspora in Paris: inventing strategies of visibility and legitimacy in a plural 'mono-cultural' society. In Gallo Ester (Ed.), Migration and Religion in Europe. Comparative perspectives on South Asian experiences, Farnham, Ashgate, Urban Anthropology series, p. 211-231.

- Goreau-Ponceaud A. et Madavan D, 2013. Dictionnaire insolite de Sri Lanka. Paris, Cosmopole, $159 \mathrm{p}$.

- Baillon, J.-F., Goreau-Ponceaud, A (Dir.), 2013, Circulations et cultures sud-asiatiques en diaspora. DESI, $\mathrm{n}^{\circ}$ 2, PUB, $213 \mathrm{p}$.

\section{ÉMILIE PONCEAUD-GOREAU}

Émilie Ponceaud-Goreau, emilie.ponceaud@etu.u-bordeaux3.fr, est doctorante en géographie, membre de l'UMR ADESS CNRS et associée au CEIAS (UMR 8564). Ses travaux portent sur la diffusion de l'enseignement pré-primaire en Inde du Sud. 